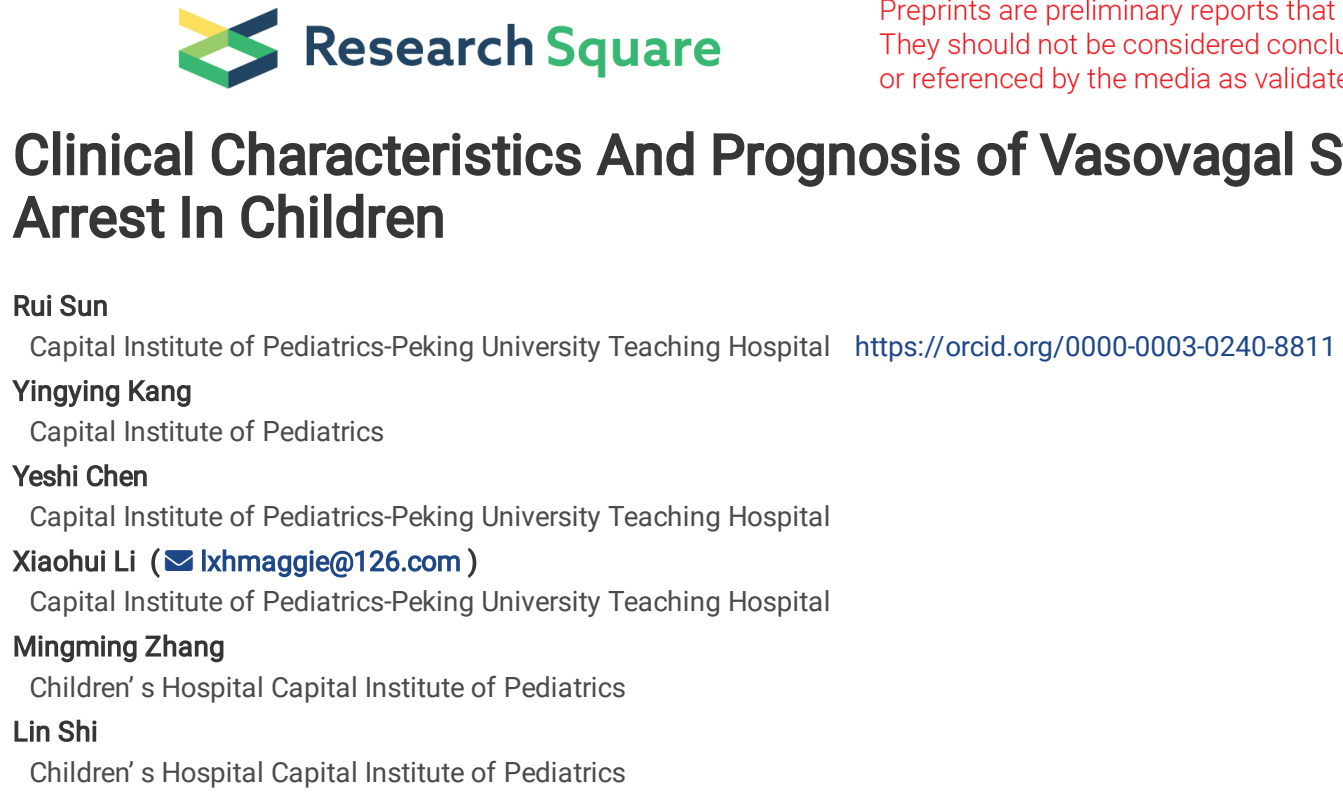

\title{
Clinical Characteristics And Prognosis of Vasovagal Syncope With Sinus Arrest In Children
}

Keywords: children, vasovagal syncope, sinus arrest,

Posted Date: December 1st, 2021

DOl: https://doi.org/10.21203/rs.3.rs-1103825/v1

License: (c) (1) This work is licensed under a Creative Commons Attribution 4.0 International License. Read Full License 


\section{Abstract}

The aim of this study was to analyze the clinical characteristics and prognosis of vasovagal syncope(VVS) with sinus arrest in children. From July 2017 to October 2020, cases of VVS with sinus arrest were recruited from patients diagnosed as VVS in our department. The clinical data, the results of the head-up tilt testing (HUTT), and follow-up information were analyzed. 223 patients were diagnosed as VVS during the time, and 10 cases of VVS with sinus arrest were recruited, who had a median age of 9.5 years $\$ interquartile range: 6.8 to 10.4 years), a gender ratio of 1:1, and median sinus arrest time of $7.7 \mathrm{~s}$ (range: 3.39 to 19s). These patients were given oral rehydration salts and orthostatic training, as well as 2 children treated with metoprolol and 5 children with sertraline hydrochloride. No child was implanted with pacemakers. During a median of 26 months of follow-up (interquartile range: 21 to 33 months), only one of the children had once syncope episode, three children had 1 4 times of prodromal symptoms. Conclusion The present research most crucial finding was that VVS patients with sinus arrest in children were younger than ordinary pediatric VVS patients. Oral rehydration salts(ORS) and autonomic nerve function exercise may improve clinical symptoms in children with VVS accompanied by sinus arrest. Pacemakers for VVS with sinus arrest in children should be cautious.

\section{What Is Known}

1. Vasovagal syncope (VVS) accounts for $70 \sim 80 \%$ of pediatric syncope cases.

2. VVS with sinus arrest in children is rare and prognosis of them is unclear.

\section{What is New}

1. VVS with sinus arrest in children were younger than ordinary pediatric VVS patients.

2. Cardiac pacemaker should be caution with pediatric neurocardiogenic syncope due to a good prognosis.

\section{Introduction}

Vasovagal syncope (VVS) accounts for 70 80\% of pediatric syncope cases. ${ }^{[1]}$ The recurring attacks of VVS accompanied by bradycardia or sinus arrest have negative effects on children's physical and mental health and significantly attenuate the quality of life for both children and parents.

VVS with sinus arrest can be seen in children with cardioinhibitory and mixed type during head-up tilt testing (HUTT), but the incidence and prognosis of such children are not clear. At present, cardiac pacing was recommended for adults diagnosed VVS with prolonged spontaneous pauses. ${ }^{[2,3]}$ But there is no clear recommendation for people aged $\varangle 40$, especially for children suffered VVS with sinus arrest. According to the Canadian statements, pacing is a highly invasive treatment in pediatric patients and should be undertaken only in consultation with a pediatric arrhythmia specialist in exceptional cases. ${ }^{[4]} 2018$ Chinese experts consensus statements suggested that implementation of cardiac pacemakers should be considered in children diagnosed with VVS with symptomatic prolonged systolic pause $₫ 4 \mathrm{~s}$, who survived from cardiopulmonary resuscitation, under the guidance of experienced pediatric cardiologists. ${ }^{[1]}$ Both the Canadian and Chinese guidelines for syncope suggested that most children with syncope have a better prognosis than adults and need to be fitted with a pacemaker with caution. ${ }^{[1,4]} 2021$ PACES expert consensus statement on the indications and management of cardiovascular implantable electronic devices in pediatric patients suggested that permanent pacing is not indicated for neurocardiogenic syncope on the basis of a positive cardioinhibitory tilt response ${ }^{[5]}$. However, the supportive evidence is limited.

There is a lack of research data on the clinical characteristics, treatment, and prognosis of children with VVS with sinus arrest currently. This study reported a few cases of VVS children with sinus arrest during syncope. The clinical data and prognosis of these children were summarized and analyzed to define the clinical characteristics and provide therapeutic experiences for VVS with sinus arrest in children.

\section{Methods}

\subsection{Study population}

From July 2017 to October 2020, the study recruited cases of VVS with sinus arrest from patients diagnosed in our department.

The diagnostic criteria, the HUTT methodology, and the positive criteria responses of HUTT are based on Guidelines for the Diagnosis of Syncope in Children.

${ }^{[6]}$ The patients with sinus arrest were detected by electrocardiogram(ECG) during HUTT or 24-hour electrocardiogram (Holter) among syncope.

\subsection{Research Method}

The result of physical examination, biochemical examination, thyroid function, ECG, Holter, echocardiography, coronary artery ultrasound, chest imaging, electroencephalogram, head CT, MRI, and the detailed history of all the cases were recorded. Of note, subjects with metabolic, cardiac, and neurologic diseases were excluded before implementation of HUTT. The body mass index(BMI) of our cases was compared with the data in the BMI graph of children aged 2 18 in China, which defined $P_{50}$ as the average growth rate value and regarded $P_{3}$ to $P_{97}$ as the normal variation range ${ }^{[7]}$.

All subjects were constantly followed up in the outpatient department or via telephone until October 2021. Meanwhile, we asked children and parents to report the pharmacotherapy patients received, whether pacemaker was implanted, and the frequency and duration of syncope after discharge.

\section{Results}




\subsection{General information}

223 children were diagnosed VVS from July 2017 to October 2020.10 patients with VVS and sinus arrest were recruited, with a median age of 9.5 years (interquartile range: 6.8 to 10.4 years). There were 5 boys and 5 girls. Clinical data of the patients are listed in Table1. Additionally, one child's grandmother died suddenly at work, one child's cousin had a history of epilepsy, and two children had a family history of syncope in their father/mother. The BMI ranged from 13.77 to $17.35 \mathrm{~kg} / \mathrm{m}^{2}$ and 1 case's $\mathrm{BMI}$ was below $\mathrm{P}_{3}$, and $9(90 \%)$ cases had normal BMI.

\subsection{Results Of Hutt}

During HUTT, $90 \%$ (9/10) of the subjects developed syncope with sinus arrest. However, sinus arrest didn't occur during the HUTT of case 3, whose heart rate decreased to 48 beats per minute. Syncope of case 3 occurred during hospitalization (while waiting for blood tests) and $3.4 \mathrm{~s}$ of sinus arrest was recorded by Holter.(Table2)

\subsection{Clinical Manifestation}

All cases had 1 10 episodes of syncope. In 9 cases (90\%), syncope appeared after characteristic prodromal symptoms such as dizziness (6/10), visual disturbances (7/10), nausea and vomiting (4/10), bellyache (1/10), and sweating (1/10). Preceding palpitations, which presented in $10 \%$ (1/10) of patients, were not usual symptoms heralding VVS. There was a significant correlation between syncope and body position: in the medical history of the subjects, syncope was primarily caused by prolonged standing position (8/10, in which 2 patients were experiencing emotional stress caused by blood draw when fainted) and a sudden change of the body position (1/10). In addition, 1/10 of the patients suffered from syncopal episodes due to emotional stress or exercise. Suspicious odor irritation was reported before 1 syncope episode in case 8 . (Table3)

In this study, the common clinical manifestations of syncope were pallor (8/10) and hypodynamia (9/10). Meanwhile, only 1 case accompanied stiffness or tremors in limbs. (Table3)

No subject had orientation obstacle or trauma when they regained consciousness. Case 1 and 3 had urinary incontinence, and $3 / 10$ of the children occurred nausea and vomiting after regaining consciousness. (Table3)

\subsection{Other Auxiliary Examination}

$3.4 \mathrm{~s}$ of sinus arrest was recorded by Holter among syncope in case3, but the results of Holter of other subjects had no obvious abnormity, while case 8 had 27 atrial premature in $24 \mathrm{~h}$, case 6 had 1 atrial premature in $24 \mathrm{~h}$, and case 9 had 70 premature ventricular in 24h. Case1, case2, case3, case7, case10 had intrasinus wandering pacemaker in Holter. Other auxiliary examinations didn't show significant abnormality. We made a thorough inspection and ruled out cardiovascular diseases, electrolyte disturbances, epilepsy, and other possible causes of VVS with sinus arrest.

\subsection{Treatment And Prognosis}

2.5.1. Health education and basic treatments All patients and their parents accepted following health education: to avoid potential triggers (e.g. prolonged standing, exposure to high temperatures, dehydration, coughing, physical pain, sudden onset of extreme emotions, etc.), to identify prodromal symptoms, and to implement physical actions that help avoid syncope. In addition, we advised children and parents to enhance communication between each other so that they can avoid overanxious or panic, and we encouraged children to do suitable exercises. Basic treatments included tilt training and oral rehydration salts (ORS). The compliance with basic treatment showed in Table4.

2.5.2. Medication Case4 was given metoprolol ( $12.5 \mathrm{mg} /$ time, q.d.) orally for 2.5 years and then self-discontinued, while case7 was given metoprolol ( 12.5 $\mathrm{mg} /$ time, b.i.d.) orally for 2 months and then self-discontinued. Sertraline hydrochloride ( $12.5 \mathrm{mg} / \mathrm{time}$, q.d.) was given to five children, among which case 3 took it for 2 months and self-discontinued; case 5 took it for 3 months and self-discontinued; case 1 was found unfocused after taking medicine and thus his parents stopped the drug; case9 stopped the drug under doctor's instructions 7 months after diagnosed due to disappearance of symptoms; and case8, being followed-up for 2.2 years, was still on medication (16.7 mg, q.d.) due to the sustained positive HUTT. (Table4)

2.5.3. Prognosis Patients were followed up for 12 to 50 months, the median follow-up period was 26 months. During the follow-up period, Case6 had 1 syncope occurred, 1 time of prodromal symptom. Case 2 had 4 times, and case 4 had 3 times of prodromal symptoms. Neither symptoms occurred in $6 / 10$ of the cases. (Table4)

\section{Discussion}

We described in detail the clinical features and prognosis of 10 children diagnosed as VVS with sinus arrest. The most crucial finding was that VVS patients with sinus arrest were younger and had good prognoses without pacemakers.

Epidemiological surveys showed that the age of onset of syncope has two peaks at teenage and $\nabla 60$ years. VVS is the most common underlying cause of neurally mediated syncope and is more prevalent in females than males. In our research, the foundation was different from above due to the younger median age of patients, and lack of gender difference. A previous study showed that the risk of syncope is inversely related to the BMI of college students and the 
morbidity of VVS significantly increases when $\mathrm{BMI}<18.5 \mathrm{~kg} / \mathrm{m}^{2}[8]$. No related study reports on the BMI characteristics of preschool and school-age children. In our study, it was showed that $90 \%$ cases' BMI was in normal range. Therefore, the physical examination of children with syncope should be noticed, and the likelihood of VVS diseases shouldn't be ignored in normal BMI children.

Since the mechanism of intra-sinus wandering pacemaker may be related to high vagal tone, the result above suggested that the vagal tone of $50 \%(5 / 10)$ cases was higher; but this finding contradicted the conclusion of Stanczyk' study ${ }^{[9]}$, in which the VVS children had increased sympathetic nerve impulses and decreased vagal tones. Even so, no significant difference was found between the baseline heart rate variability (HRV) of asymptomatic VVS patients and healthy children ${ }^{[10]}$. Previous studies had shown that HRV varies with age and is related to sex and race ${ }^{[11,12]}$, but there is no international recognized reference value. Further studies concerning the difference in autonomic nerve function between VVS patients with or without sinus arrest under rest stage are still needed.

In our study, premonitory signs and symptoms were consistent with previous studies among pediatric VVS patients. The ECG, the cardiac functions, and the lung functions were normal, despite the chest tightness as the main precursor in case 5 and the repeated palpitation during syncope in case 10 . Thus, it seems that children with chest tightness and palpitations should also be distinguished from VVS. Nausea and vomiting was presented before losing consciousness in four cases and after recovery of consciousness in three cases. This clinical feature is consistent with the results of Heyer's studies, which stated that the gastric dysrhythmia during tilt-induced syncope consisted of a high amplitude bradygastria with a tachygastria component that began well before syncope and continued for several minutes during recumbent recovery, followed by attenuation of amplitudes. This is because the nausea is temporally associated with changes in gastric myoelectrical activity as well as increased serum concentrations of vasopressin and epinephrine. ${ }^{[13]}$ The time of losing consciousness in this study was recorded mainly based on the information provided by witnesses, but this information may be inaccurate, because the onset of syncope only lasts for several seconds, and in a stressful state, the time of losing consciousness is usually described as the time of the entire syncopal episode. Children who did not provide detailed information may be associated with a short period of syncope, which wasn't observed or valued.

As for treatment, all children were given health education and basic treatments. But not all children had good patient compliance. In this paper, the children's drug treatment referred to the clinical studies for recurrent syncope. A trial in adults showed that metoprolol is effective for preventing recurrence of malignant VVS in adults ${ }^{[14]}$. Although metoprolol may be effective in children ${ }^{[15]}$, the mechanisms of action of metoprolol in malignant VVS in children and the prognosis of treated patients remain unclear. In our study, 2 of 10 cases took metoprolol, and one of them occurred prodromal symptoms during the follow-up period. In addition, a previous study in our center indicated that platelet 5-hydroxytryptamine(5-HT) is higher in HUTT-positive VVS children, which suggested that the central serotonergic system might be involved in the pathogenesis of VVS. Sertraline hydrochloride was proved to be effective in preventing recurrent neurocardiogenic syncope in children with refractory VVS with recurrent syncope. Likewise, a previous trial showed that selective serotonin reuptake inhibitors (SSRIs) were effective and well-tolerated in the treatment of recurrent refractory VVS in adults ${ }^{[16]}$. Also, adult depression and anxiety are proved to be independent risk factors for VVS patients ${ }^{[17]}$, which provided a rationale for using SSRIs in the treatment of VVS. Even so, the effectiveness of SSRIs in children with malignant VVS is still uncertain. In this study, 5 of 10 cases took sertraline hydrochloride, no one developed syncope during the follow-up period. Lastly, studies on adult malignant VVS proved that pacemakers are effective in elderly patients. A recent study found that in patients aged 40 years or older, who are affected by severe recurrent vasovagal syncope and tilt-induced asystole, dual-chamber pacemaker with closed-loop stimulation(CLS) is highly effective in reducing the recurrences of syncope. ${ }^{[18]}$ Studies on the treatment of VVS children with cardiac arrest remain elusive, we found only two reports on the use of pacemakers in children with neurally mediated syncope complicated by sinus arrest. The number of cases of the two articles was 12 and 11 , respectively. In the study of 12 children aged 2 14 years (median age: 2.8 years), patients had 10 40s sinus arrest in cardiac monitoring and the symptoms improved after pacemaker installation ${ }^{[19]}$; in the other study about 11 pediatric patients (0.8 17 years, median age: 2.7 years) with sinus arrest(11.2 21s), cardiac pacing also reduced syncope episodes ${ }^{[20]}$. Both studies showed that the installation of a pacemaker reduces the incidence of neuro-mediated syncope with sinus arrest in children. ${ }^{[18,19]}$ But neither of these studies classified syncope, and VVS children with sinus arrest were not analyzed. In a case report of a 17-year-old boy with severe cardioinhibitory VVS, the installation of a pacemaker reduced the incidence of syncope. ${ }^{[21]}$ In another case report, however, a 16-year-old boy had recurrent syncope caused by blood-injury phobia, which was improved with cognitive behavioral therapy and did not require a pacemaker. ${ }^{[22]}$ In the current study, children diagnosed VVS and sinus arrest were included without pacemaker installation, and only one child has once syncope occurred during the follow-up period. Based on the evidence above, pacemakers should be carefully installed in syncope patients with incomplete autonomic nerve development and children with VVS with sinus arrest.

Of note, case 10 had an early age of onset and was misdiagnosed as epilepsy due to the presence of stiffness of the limbs during syncopal episodes and the occasional trembling of the limbs; these symptoms were not improved by 6 years of anti-epileptic treatment. The patient was diagnosed as malignant VVS after implementation of HUTT. Therefore, children with epilepsy who respond poorly to drug treatments should be recommended to use HUTT to identify VVS. Also, attention should be paid to the differential diagnosis of children with syncope at a young age of onset. Further research is warranted to strengthen our understanding of underlying mechanisms of VVS, which may be associated with abnormal Bezold-Jarish reflex, dysfunction of the autonomic nervous system, neuro-humoral factors, etc.

Limitations of the study include the small number of patients and the short time follow-up. In addition, the present study was retrospective and observational, and the medical treatments of cases were different. Consequently, the efficacy of drugs on prognosis needs to be studied further by randomized controlled trials.

\section{Conclusions}

The study suggested that the children with VVS with sinus arrest during syncope were younger than ordinary pediatric VVS patients, and there was no significant difference in the clinical manifestations between them. Moreover, the result of this study demonstrated that the regular consumption of ORS and 
autonomic nerve function exercises may be responsible for the good prognosis by far. The relatively good prognosis also suggests that pacemakers are worth studied in children with VVS with sinus arrest.

\section{Abbreviations And Acronyms}

$\mathrm{AV}=$ atrioventricular

BMI=Body Mass Index

Holter=24-hour electrocardiogram

HRV=Heart rate variability

HUTT=Head-up tilt test

ORS $=$ oral rehydration salts

SSRIs=selective serotonin reuptake inhibitors

VVS=Vasovagal syncope

\section{Declarations}

Funding Source. This work was supported by Beijing Hospital Administration “Peak Climbing” Talents Program (NO.DFL20181301), XHL

Conflict of interest. The Authors declare that there is no conflict of interest.

Availability of data and material. The data underlying this article are available in the article.

Code availability. Not applicable

Author Contributors $₫ \mathrm{XL}$ designed the study. RS and YK collected and analysed the data. RS drafted the manuscript and $\mathrm{YC}$ revised the paper. $\mathrm{XL}, \mathrm{MZ}$ and $\mathrm{LS}$ contributed to the interpretation of the results and critical revision of the manuscript.

Ethics approval: This study was approved by the Ethics Committee of Capital Pediatric Research Institute (SHERLLM2021025).

Consent to participate. Informed consent was obtained from all individual participants included in the study.

Consent for publication: All authors agreed to submit our manuscript to this journal.

\section{References}

1. Liao Y, Du JB (2018) Expert consensus on the treatment of vasovagal syncope and postural tachycardia syndrome in children[J]. Chin J Pediatr 56(1):1012. DOI:10.3760/cma.j.issn.0578-1310.2018.01.003

2. Shen WK, Sheldon RS, Benditt DG, Cohen MI, Forman DE, Goldberger ZD, Grubb BP, Hamdan MH, Krahn AD, Link MS et al (2017) 2017 ACC/AHA/HRS guideline for the evaluation and management of patients with syncope: Executive summary: A report of the American College of Cardiology/American Heart Association Task Force on Clinical Practice Guidelines and the Heart Rhythm Society[J]. Heart Rhythm 14(8):e218-e254. DOl: 10.1161/CIR.0000000000000498

3. Michele Brignole ÁMFJ, Alessandra Fanciulli A F R F, Martín VPMJ, Van Dijk G (2018) 2018 ESC Guidelines for the diagnosis and management of syncope[J]. Eur Heart J 71(10):1883-1948. DOI: 10.1093/eurheartj/ehy037

4. Sanatani SMF, Chau VMF, Fournier AMF, Dixon AMF, Blondin RM, Sheldon RSMF (2016) Canadian Cardiovascular Society and Canadian Pediatric Cardiology Association Position Statement on the Approach to Syncope in the Pediatric Patient[J]. Can J Cardiol 33(2):189-198. DOI: 10.1016/j.cjca.2016.09.006

5. Shan MJ, Silka MJ, Avari SJ, Balaji S, Beach CM, Benjamin MN, Berul Cl, Cannon B, Cecchin F, Cohen MI (2021) 2021 PACES expert consensus statement on the indications and management of cardiovascular implantable electronic devices in pediatric patients[J]. Indian Pacing Electrophysiol J 21(6):367393. DOI: 10.1016/j.ipej.2021.07.005

6. Cardiovascular, Section, Chinese Society of Pediatrics. Guidelines for the diagnosis of syncope in children(2016)[J].Chin J Pediatr,2016, 54(4):246-250. DOI: 10.3760/cma.j.issn.0578-1310.2016.04.003

7. Zong XN, Li H (2009) General growth patterns and simple mathematic models of height and weight of Chinese children[J].Chin J Pediatr, (05):371-375

8. Yamada T, Yanagimoto S (2017) Dose-Response Relationship between the Risk of Vasovagal Syncope and Body Mass Index or Systolic Blood Pressure in Young Adults Undergoing Blood Tests[J]. Neuroepidemiology 49(1-2):31-33. DOI: 10.1159/000479698

9. Zygmunt A, Stanczyk J (2004) Heart rate variability in children with neurocardiogenic syncope[J]. Clin Auton Res 14(2):99-106. DOI: 10.1007/s10286004-0168-0 
10. Akcaboy M, Atalay S, Ucar T, TUTAR E (2011) Heart rate variability during asymptomatic periods in children with recurrent neurocardiogenic syncope[J]. Turk J Pediatr 53(1):59-66

11. van den Berg ME, Rijnbeek PR, Niemeijer MN, Hofman A, Van HG, Bots ML, Hillege H, Swenne CA, Eijgelsheim M, Stricker BH et al (2018) Normal Values of Corrected Heart-Rate Variability in 10-Second Electrocardiograms for All Ages[J]. Front Physiol 9(424):1-9. D0I: 10.3389/fphys.2018.00424

12. Koenig J, Thayer JF (2016) Sex differences in healthy human heart rate variability: A meta-analysis[J]. Neuroscience \& Biobehavioral Reviews 64:288310. DOI: 10.1016/j.neubiorev.2016.03.007

13. Heyer GL, Boles LH, Harvey RA, Cismowski MJ (2018) Gastric myoelectrical and neurohormonal changes associated with nausea during tilt-induced syncope[J]. Neurogastroenterology \& Motility 30(3):e13220. DOI: 10.1111/nmo.13220

14. Mauro Biffi GBPS, Romano Zannoli (1997) A B B M. Malignant vasovagal syncope a randomised trial of metoprolol and clonidine[J]. Heart 77:268-272. DOI: $10.1136 /$ hrt.77.3.268

15. Kremastinos DT (2008) Cardiogenic syncope and serotonin reuptake inhibitors[J]. Hellenic J Cardiol 49(5):375-376

16. Di Girolamo E, Di lorio C, Sabatini P, Leonzio L, Barbone C, Barsotti A (1999) Effects of paroxetine hydrochloride, a selective serotonin reuptake inhibitor, on refractory vasovagal syncope: a randomized, double-blind, placebo-controlled study[J]. J Am Coll Cardiol 33(5):1227-1230. DOI: 10.1016/s07351097(98)00694-9

17. Atici A (2020) The Relationship Between Clinical Characteristics and Psychological Status and Quality of Life in Patients with Vasovagal Syncope[J]. Northern Clinics of Istanbul 7(3):237-245. DOI: 10.14744/nci.2020.93753

18. Brignole M, Russo V, Arabia F, Oliveira M, Pedrote A, Aerts A, Rapacciuolo A, Boveda S, Deharo CJ, Maglia G et al (2021) Cardiac pacing in severe recurrent reflex syncope and tilt-induced asystole[J]. Eur Heart J 42(5):508-516. Cardiac pacing in severe recurrent reflex syncope and tilt-induced asystole

19. McLeod KA, Wilson N, Hewitt J, Norrie J, Stephenson JB (1999) Cardiac pacing for severe childhood neurally mediated syncope with reflex anoxic seizures[J]. Heart 82(6):721-725. DOI: 10.1136/hrt.82.6.721

20. Paech C, Wagner F, Mensch S, Antonin GR (2018) Cardiac pacing in cardioinhibitory syncope in children[J]. Congenit Heart Dis 13(6):1064-1068. DOI: $10.1111 /$ chd. 12682

21. De Regibus V, Moran D, Chierchia GB, Brugada P, De Asmundis C (2018) Leadless pacing in a young patient with cardioinhibitory vasovagal syncope[J]. Indian Pacing Electrophys J 18(3):120-122. DOI: 10.1016/j.ipej.2017.12.003

22. Van Dijk N, Velzeboer SC, Destree-Vonk A, Linzer M, Wieling W (2001) Psychological treatment of malignant vasovagal syncope due to bloodphobia[J]. Pacing Clin Electrophysiol 24(1):122-124. DOI: 10.1046/j.1460-9592.2001.00122.x

\section{Tables}

Table1 Basic information of 10 cases of vasovagal syncope with sinus arrest

\begin{tabular}{|c|c|c|c|c|c|c|c|c|c|}
\hline \multirow[t]{3}{*}{ Patient } & \multirow{3}{*}{ Gender } & \multirow[t]{3}{*}{ Age(year) } & \multirow[t]{3}{*}{$\mathrm{BM} \otimes \mathrm{kg} / \mathrm{m}^{\wedge} 2 \rrbracket$} & \multirow[t]{3}{*}{ Course } & \multirow[t]{3}{*}{ Sycope episode } & \multirow[t]{3}{*}{ previous history } & \multicolumn{3}{|c|}{ family history } \\
\hline & & & & & & & sudden & neurological disorders & syncope \\
\hline & & & & & & & death & & \\
\hline Case1 & girl & 6.25 & 15.12 & 2 years & 1 & health & - & - & - \\
\hline Case2 & girl & 8.33 & 16.32 & 2years & 1 & health & + & - & - \\
\hline Case3 & girl & 9.50 & 16.09 & 7years & 7 & health & - & - & - \\
\hline Case4 & girl & 9.75 & 15.75 & 4years & 6 & health & - & + & - \\
\hline Case5 & girl & 10.33 & 17.35 & 3years & 5 & health & - & - & - \\
\hline Case6 & boy & 6.42 & 15.75 & 2years & 4 & health & - & - & - \\
\hline Case7 & boy & 6.92 & 15.67 & 9days & 4 & health & - & - & - \\
\hline Case8 & boy & 9.58 & 16.65 & 4years & 4 & health & - & - & - \\
\hline Case9 & boy & 10.42 & 15.41 & 8days & 1 & health & - & - & + \\
\hline Case10 & boy & 11.58 & 13.77 & 7years & 10 & health & - & - & + \\
\hline
\end{tabular}

+: positive family history ;:- negative family history

Course: the time from the first syncope to diagnosis of VVS with sinus arrest in our department

Table2 The results of HUTT in children with vasovagal syncope with sinus arrest 


\begin{tabular}{|c|c|c|c|c|c|}
\hline Patient & $\begin{array}{l}\text { The time of } \\
\text { onset(min) }\end{array}$ & $\begin{array}{l}\text { Sinus arrest time } \\
\text { (s) }\end{array}$ & Type & Clinical manifestation during HUTT & $\begin{array}{l}\text { Nitroglycerin during } \\
\text { HUTT }\end{array}$ \\
\hline Case1 & 45 & 5.98 & 1 & Pallor, hypodynamia, sweating & + \\
\hline Case2 & 18 & 6 & 2 & $\begin{array}{l}\text { Pallor,circumoral cyanosis, chest } \\
\text { distress,nausea,aconuresis }\end{array}$ & - \\
\hline Case3 & 25 & / & 2 & Pallor,dizziness,chest distress & - \\
\hline Case4 & 45 & 12 & 1 & Pallor,dizziness,sweating & + \\
\hline Case 5 & 45 & 19 & 2 & Pallor & + \\
\hline Case6 & 45 & 10 & 2 & Sweating,aconuresis & + \\
\hline Case7 & 50 & 3.39 & 1 & Pallor,nausea & + \\
\hline Case8 & 25 & 9.4 & 2 & Pallor,chest distress,sweating,amaurosis & - \\
\hline Case9 & 45 & 11 & 2 & Pallor,dizziness & + \\
\hline Case10 & 20 & 5.44 & 2 & Pallor,dizziness,tic & - \\
\hline
\end{tabular}

HUTT囚head-up tilt testing

The time of onset: from the start of the HUTT test to the onset of syncope

Type: the positive response to HUTT:type 1 (mixed), type 2 (cardioinhibitory), and type 3 (vasodepressor).

+:with nitroglycerin provocation during HUTT

-:without nitroglycerin provocation during HUTT

/:without syncope during HUTT

Table3 Clinical manifestations of children with vasovagal syncope with sinus arrest 


\begin{tabular}{|c|c|c|c|c|c|c|c|c|c|c|}
\hline Patient & Case1 & Case2 & Case3 & Case4 & Case5 & Case6 & Case7 & Case8 & Case9 & Case10 \\
\hline \multicolumn{11}{|c|}{ Circumstances of syncope } \\
\hline Prolonged standing & + & + & + & - & + & - & + & + & + & + \\
\hline Posture changes & - & - & + & + & - & - & - & - & - & - \\
\hline Emotional stress & - & + & - & - & - & + & - & - & - & + \\
\hline Post-exercise & - & - & - & - & - & + & - & - & - & - \\
\hline Unpleasant odor & - & - & - & - & - & - & - & + & - & - \\
\hline \multicolumn{11}{|c|}{ Prodromal symptoms } \\
\hline Chest distress & - & - & - & - & + & - & - & - & - & - \\
\hline Palpitations & - & - & - & - & - & - & - & - & - & + \\
\hline Dizziness & + & + & + & - & - & + & + & + & + & - \\
\hline amaurosis & + & + & + & - & - & - & + & + & - & + \\
\hline Nausea and vomit & + & + & - & - & - & + & + & - & - & - \\
\hline abdominal pain & + & - & - & - & - & - & - & - & - & + \\
\hline Sweating & - & - & - & - & - & - & - & - & + & - \\
\hline Vision disorder & - & - & - & - & - & - & - & - & + & - \\
\hline \multicolumn{11}{|l|}{ During episode } \\
\hline Pale skin & + & + & + & + & + & \pm & - & + & + & / \\
\hline Weakness & + & + & + & + & + & + & + & + & + & - \\
\hline Tremors in the limbs & - & - & - & - & - & - & - & - & - & + \\
\hline \multicolumn{11}{|l|}{ After episode } \\
\hline disorientation & - & - & - & - & - & - & - & - & - & - \\
\hline trauma & - & - & - & - & - & - & - & - & - & - \\
\hline aconuresis & + & - & \pm & - & - & - & - & - & - & - \\
\hline Nausea and vomit & - & + & - & - & - & - & + & - & + & - \\
\hline
\end{tabular}

$\therefore$ : existing $\varangle$ : nonexistent $₫ \pm$ : occasional $\varangle /$ : unknowing

Table4 Treatment and Prognosis of children with vasovagal syncope with sinus arrest

\begin{tabular}{|c|c|c|c|c|c|c|c|c|c|c|}
\hline \multirow[t]{2}{*}{ Patient } & \multirow[t]{2}{*}{ ORS,m } & \multirow{2}{*}{$\begin{array}{l}\text { Regular } \\
\text { title } \\
\text { traing, } \\
\mathrm{m}\end{array}$} & \multicolumn{2}{|l|}{ Medicine } & Follow-up 3 months & \multicolumn{2}{|c|}{ Follow-up 1 year } & \multicolumn{2}{|c|}{ Follow-up 2 years } & Follow-u| \\
\hline & & & $\begin{array}{l}\text { Sertraline } \\
\text { hydrochloride,m }\end{array}$ & metoprolol,m & $\begin{array}{l}\text { prodromal } \\
\text { symptoms }\end{array}$ & syncope & $\begin{array}{l}\text { prodromal } \\
\text { symptoms }\end{array}$ & syncope & $\begin{array}{l}\text { prodromal } \\
\text { symptoms }\end{array}$ & syncope \\
\hline
\end{tabular}

\begin{tabular}{|c|c|c|c|c|c|c|c|c|c|c|c|}
\hline Case1 & 3 & 12 & 3 & - & 0 & 0 & 0 & 0 & & & \\
\hline Case2 & 2 & 30 & - & - & 0 & 0 & 0 & 0 & 0 & 2 & 0 \\
\hline Case3 & 2 & 2 & 2 & - & 0 & 0 & 0 & 0 & 0 & 0 & \\
\hline Case4 & 25 & 2 & - & 30 & 0 & 0 & 0 & 0 & 0 & 2 & 0 \\
\hline Case5 & 3 & 25 & 3 & - & 0 & 0 & 0 & 0 & 0 & 0 & \\
\hline Case6 & 15 & 30 & - & - & 0 & 0 & 0 & 1 & 1 & 0 & 0 \\
\hline Case7 & 2 & 2 & - & 2 & 0 & 0 & 0 & 0 & & & \\
\hline Case8 & 26 & 26 & 26 & - & 0 & 0 & 0 & 0 & 0 & 0 & \\
\hline Case9 & 26 & 13 & 8 & - & 0 & 2 & 0 & 0 & 0 & 0 & \\
\hline Case10 & 30 & 30 & - & - & 0 & 0 & 0 & 0 & 0 & 0 & 0 \\
\hline
\end{tabular}

